\title{
TTR
}

Traduction, terminologie, rédaction

\section{Présentation : la banlieue française au prisme de la traduction littéraire}

\section{Ilaria Vitali}

Volume 31, numéro 1, 1er semestre 2018

Traduire la banlieue : problématiques, enjeux, perspectives

Translating the Banlieue: Issues, Challenges, Perspectives

URI : https://id.erudit.org/iderudit/1062544ar

DOI : https://doi.org/10.7202/1062544ar

Aller au sommaire du numéro

\section{Éditeur(s)}

Association canadienne de traductologie

\section{ISSN}

0835-8443 (imprimé)

1708-2188 (numérique)

Découvrir la revue

Citer ce document

Vitali, I. (2018). Présentation : la banlieue française au prisme de la traduction littéraire. TTR, 31(1), 9-21. https://doi.org/10.7202/1062544ar d'utilisation que vous pouvez consulter en ligne. 


\title{
Présentation : la banlieue française au prisme de la traduction littéraire
}

\author{
Ilaria Vitali \\ Università di Macerata
}

Comment les banlieues françaises sont-elles perçues hors de France, et dans quelle mesure la traduction littéraire façonne-telle leur image? De prime abord, lorsqu'on pense aux banlieues, des images liées à la violence urbaine nous viennent à l'esprit, sans aucun doute à cause des émeutes des années 2000 et de la dramatisation médiatique qui les a accompagnées (Vieillard-Baron, $2008)^{1}$. Or, si l'on prend le temps d'observer de plus près l'objet d'étude qu'est la banlieue, on s'aperçoit que le spectre est bien plus large que ce que l'on pourrait croire. Le mot «banlieue» lui-même est polysémique et porteur de significations multiples. Ce n'est pas un hasard si Thierry Paquot (2008) le définit comme un «singulier pluriel». Létymologie du mot nous dit qu'à l'origine, il appartenait au domaine juridique, plus précisément au droit féodal; la banlieue désignait alors un territoire annulaire de l'ampleur d'une lieue (environ 4 kilomètres) situé autour du domaine seigneurial où s'exerçait le droit de ban. Aujourd'hui, cependant, le mot «banlieue» a été re-sémantisé par de nombreux écrivains et artistes qui le reconduisent à l'ancienne pratique de la mise au ban, en soulignant la forte tendance ghettoïsante qui est à la base de l'organisation architectonique, sociale et culturelle de ces espaces suburbains. Ce

1. La question des émeutes en banlieue n'est pas nouvelle; elle secoue les périphéries françaises depuis la fin des années 1970. Cependant, les révoltes des années 2000 ont bénéficié d'une couverture médiatique exceptionnelle qui a eu des répercussions sociales, culturelles et politiques. La crise des banlieues fait l'objet de nombreuses études : comme le rappelle Bernard Wallon (2016, p. 9), le site de la Documentation française recense plus de cent travaux depuis 1995. Kokoreff et Lapeyronnie (2013), Hargreaves (2016) et Wallon (2016), entre autres, proposent des réflexions récentes sur le sujet. 
glissement montre l'ampleur sémantique du terme qui, selon Hervé Vieillard-Baron, géographe et spécialiste de la banlieue, recouvre au moins cinq notions :

une notion juridique se rapportant au droit féodal, une notion géographique, celle de ceinture urbanisée dépendante du centre, une notion sociologique rendant compte de l'exclusion qui touche les habitants des marges urbaines, une notion culturelle qui fait référence aux pratiques festives qui sont nées sur ce territoire et qui y sont associées (tags, rap, festivals comme celui de Banlieue bleu en Seine-Saint-Denis...), et enfin une notion symbolique pour exprimer le discrédit qui pèse sur une partie des populations résidentes. (2008, p. 22)

La banlieue, une et plusieurs, se prête à des lectures multiples, et «le pouvoir évocateur du mot change considérablement d'une époque à l'autre» (ibid., p. 31). D'après Vieillard-Baron, c'est à la fin du XIX ${ }^{\mathrm{e}}$ siècle que s'enracine l'image de la banlieue contemporaine, perçue d'une manière dépréciative, lorsque se crée un système de valeurs qui oppose Paris à la province. La banlieue de Paris est en effet la première de France, chronologiquement et quantitativement. $\mathrm{Au}$ cours du XXe siècle, la banlieue évolue encore, les usines et les logements HLM se multiplient, et l'image qu'elle renvoie devient progressivement celle des grands ensembles des cités, avec leurs batteries de barres et de tours, au point que les mots «banlieue» et «cité» sont devenus quasiment synonymes non seulement pour les médias, mais aussi pour les écrivains et artistes, qui encouragent d'une certaine manière l'amalgame sémantique.

Conçues d'abord pour accueillir la main-d'œuvre française, les cités ont été témoins de différentes vagues d'immigration. Elles sont désormais habitées surtout par ceux que j'ai définis ailleurs comme des «intrangers»(Vitali, 2011), des Français d'origines autres, issus de l'immigration notamment maghrébine et africaine, qu'on pourrait appeler «nouveaux Français» et qui revendiquent des appartenances multiples, même si leur voix demeure peu entendue. Ce n'est pas un hasard si plusieurs chercheurs perçoivent une relation entre banlieue et (post)colonie, au point de voir dans la périphérie française la reproduction périlleuse d'un «théâtre colonial» (Lapeyronnie, 2005) ${ }^{2}$, avec une explicitation des rapports

2. Une analyse de la presse française lors des émeutes de 2005 menée par Valérie Haas et Capucine Vermande (2010) montre par ailleurs le recours massif à des images issues de l'idéologie coloniale dans les représentations des émeutiers. 
de force entre «dominant» et «dominé», et, dans le contexte littéraire, entre une littérature majeure et une littérature mineure, pour reprendre les termes de Deleuze et Guattari (1975)3.

Dans Le pays de la littérature (2003), Pierre Lepape souligne qu'en France plus qu'ailleurs politique et création littéraire sont intimement liées depuis les Serments de Strasbourg. Mais que se passe-t-il quand, dans un pays fortement centralisé comme l'Hexagone, politique et pouvoir ne sont plus (d)écrits par les écrivains issus d'un «centre» symbolique, mais plutôt par ceux qui se placent à titres divers (ethnique, social, culturel) dans les "marges», en les transformant en nouveaux «centres» de création? Les écrivains ont pris en charge les préoccupations de ces territoires situés aux écarts de la ville et de la société française, et ils l'ont fait avec les moyens propres de l'écriture. Chacun a choisi de se positionner différemment sur l'échiquier littéraire, tout en manipulant et en hybridant ses balises ${ }^{4}$. Certains ont adhéré à une littérature ouvertement impliquée dans le social et dans le politique - on se souvient du collectif Qui fait la France?, fondé en 2007, guidé par un véritable manifeste qui récusait tout purisme esthétique et toute posture initiée, et proclamait la participation active au débat public. D'autres ont préféré un «engagement oblique», forme plus subtile et intime d'implication qui engage d'abord le rapport de l'écrivain au style et à l'écriture; on pense entre autres à Rachid Djaïdani et Mabrouck Rachedi. Un élément demeure toutefois inchangé : l'enracinement dans le terrain et sa revendication. Djaïdani définit ainsi ce fort sentiment identitaire en parlant des héros de son roman Viscéral:

[p] our tout l'or du monde jamais ils n'abandonneraient leur cité téci tess, ils la revendiquent dans leur façon de respirer, de penser, de la raconter. La tess est la fondation de ce qu'ils sont, plutôt mourir que la quitter. $(2007$, p. 8$)$

3. Pour une étude récente autour de ces sujets, voir Reeck et Kleppinger (2018). Une relation ou filiation plus ou moins directe, plus ou moins nuancée, entre la littérature des banlieues et la littérature beure est également mise en relief par les chercheurs. Pour mieux cerner ces aspects, voir, entre autres, Puig (2011), Reeck (2011), Hargreaves (2014), Vitali (2014) et Horvath (2016).

4. Je ne m'attarderai pas sur le débat autour des classements et des étiquettes. J'entends ici par «littérature de banlieue» l'ensemble des fictions littéraires dont la banlieue constitue non seulement le cadre de l'action, mais également le thème principal de l'intrigue. Sur ce sujet, on peut consulter Hargreaves (2014), Horvath (2016), Reeck (2011) et Vitali (2011 et 2014). 
Tel que David Lepoutre le montrait en 1997 :

quelle que soit l'image négative dont ces lieux sont affublés, et indépendamment des difficultés éprouvées et des stratégies mises en œuvre pour assumer cette image, les cités de banlieue sont également, si étonnant que cela puisse paraître, des lieux de naissance, d'enfance et de souvenirs, objets de fantasmes et d'imaginaire pour beaucoup d'adolescents. (p. 51-52)

Les émeutes incendiaires qui ont secoué les banlieues parisiennes à l'automne 2005 et qui se sont répétées en 2006 et en 2007 ont propulsé le débat sur les périphéries urbaines au niveau médiatique dans le monde entier. En fait, il ne s'agit là que de la pointe de l'iceberg, car ces émeutes sont les derniers éléments visibles d'une révolte souvent latente qui a commencé vers la fin des années 1970, lorsque les premiers mouvements pour la reconnaissance des droits de ces territoires en souffrance, plus ou moins provocateurs, plus ou moins pacifiques, sont apparus. Les révoltes qui ont touché la banlieue lyonnaise au début des années 1980 ont déclenché, on s'en souviendra, la célèbre Marche pour l'égalité et contre le racisme de 1983, dont le trentenaire a été commémoré en $2013^{6}$.

Ces problématiques se reflètent dans la fiction littéraire, car les écrivains posent la question de la relation de la banlieue au social et au culturel, et interrogent la manière dont l'œuvre littéraire négocie cette relation. C'est sans doute en considérant les différentes modulations entre des postures à la fois polaires et complémentaires que Paquot décrit la culture des banlieues, dans son ensemble, comme une «culture-action», qui devient une "culture-création» (2008, p. 2). En effet, la banlieue, plus que d'autres espaces urbains contemporains, est traversée par de nouvelles tendances littéraires, par des productions culturelles inédites, par des créations qui sont aussi linguistiques, car les banlieues sont le berceau de ce parler étudié par les sociolinguistes et les argotologues, que Jean-Pierre Goudaillier définit comme le "français contemporain des cités", et dont beaucoup de mots et d'expressions investissent désormais la «langue des

5. L'image ambivalente de la cité, qui devient alternativement «cage» dont on voudrait s'évader et «cocon» qui protège, a aussi été soulignée par Kokoreff et Lapeyronnie (2013, p. 29-30).

6. La revue Africultures (2014) y a consacré un riche dossier, intitulé La marche en héritage (1983-2013). 
jeunes», voire le français tout court ${ }^{7}$. Jean-Michel Kasbarian notait déjà en 1997 que la banlieue est «un ensemble ouvert, instable de réalisations linguistiques ", caractérisé par la présence importante d'une dimension diastratique et surtout diatopique due à l'impact de l'immigration. Aujourd'hui, les écrivains puisent de plus en plus dans ce répertoire vivant, métissé et toujours changeant, qu'ils exploitent de manière créative dans leurs textes pour remettre en question aussi bien la langue écrite que l'institution littéraire française. En effet, le français des cités a fait l'objet d'une véritable élaboration scripturaire et poétique, au point de devenir, au cours des dernières décennies, un moyen d'écriture exploité en tout état de cause dans les romans qui ont au cœur la banlieue (Vitali, 2015). À la lumière de ces réflexions, la banlieue apparaît comme un lieu idéal pour examiner non seulement les enjeux de la France contemporaine et la manière dont ces enjeux sont pris en charge par les écrivains, mais surtout la façon dont ils dépassent les frontières nationales par le biais de la traduction. C'est justement vers ces problématiques que nous allons tourner notre regard dans ce numéro de TTR.

Au fil des années, les œuvres littéraires mettant en scène «la zone» se sont multipliées ${ }^{8}$ et, grâce à un marché de l'édition de plus en plus large et mondialisé, elles ont dépassé les frontières françaises. La périphérie, par le biais de sa représentation fictionnelle, est devenue visible et présente hors de l'Hexagone. Mais de quelle manière le transfert s'opère-t-il? Comme on l'a vu, de par leur spécificité, ces ouvrages de fiction mobilisent des rapports de force d'ordre linguistique, sociopolitique et culturel qui déterminent aussi les choix éditoriaux ainsi que les postures des traducteurs. Dans une étude de 2012, Gisèle Sapiro mettait l'accent sur les obstacles auxquels doivent faire face ces derniers. En effet, si l'on parle souvent de manière enchantée (et parfois naïve) du «dialogue entre les cultures" prôné par la traduction littéraire, on s'intéresse moins aux conditions et aux obstacles que ce dialogue peut poser. La question est pourtant primordiale, surtout lorsqu'on étudie la traduction de romans qui traitent un sujet sensible comme celui de la banlieue française. Le traducteur devra faire ses choix non seule-

7. Sur la langue des cités et ses rapports avec le français populaire et argotique, on peut se reporter à Bertucci et Delas (2004), et surtout à Gadet (2017).

8. Horvath (2016) signale plus de cent ouvrages littéraires depuis 2000. 
ment en fonction d'enjeux éthiques et idéologiques, mais également du contexte éditorial spécifique dans lequel il agira. La traduction faisant partie de ce polysystème littéraire postulé par Even-Zohar dès les années 1970 et développé par la suite (Even-Zohar, 1990; Toury, 1995 entre autres), le traducteur réel ne pourra pas faire abstraction du marché éditorial ainsi que de l'ensemble des textes originaux et des traductions déjà présents dans sa culture cible. Les macro et micro-stratégies traductives qu'il choisira ne prendront donc de sens qu'en relation à un contexte auquel il s'adaptera et qu'il contribuera en même temps à façonner et à transformer par le biais de sa traduction, comme un artisan qui ajoute une pièce à une mosaïque préexistante. Son apport sera d'autant plus important que les textes faisant partie de notre champ de recherche souhaitent remettre en cause la dichotomie centre-périphérie.

Les enjeux posés par les auteurs dont on a discuté plus haut influencent donc à leur tour l'espace traductionnel ${ }^{9}$. Comme le notait Vieillard-Baron (1996), l'emploi même du terme banlieue dans les langues étrangères ne peut se faire sans interrogation critique. Si le mot est en soi problématique, il faut bien reconnaître que les difficultés de traduction ne se limitent pas à celui-ci, mais engagent la réalité qu'il représente. Comment traduire un concept, des images et un langage si particuliers? Si l'on considère, avec Bourdieu, que les textes «circulent sans leur contexte»(2002, p. 4), on se rend vite compte que toute traduction peut engendrer des changements de perspective, voire des méprises et des malentendus. Une fois insérées dans un nouveau contexte, les traductions de romans traitant des banlieues peuvent se heurter à l'incompréhension ou à la mécompréhension, ce qui peut produire un échec ou des difficultés dans la circulation aussi bien des textes que des idées qu'ils véhiculent. La banlieue étant souvent affublée de généralisations et de clichés, il devient nécessaire de poser un regard critique non seulement sur les traductions, mais également sur leur réception, sur leur impact sur l'imaginaire culturel et social, pour voir de quelle manière les ouvrages s'insèrent dans un nouveau contexte et s'ils confortent ou corrigent les idées reçues ${ }^{10}$. S'ouvre

9. Sur les rapports entre traduction et engagement, on lira avec profit le numéro de TTR dirigé par Sherry Simon (2005), consacré à la «traduction engagée ».

10. Voir Hargreaves (2016), notamment, sur l'amalgame entre banlieue, islam et islamisme; on se souviendra, par ailleurs, qu'aux États-Unis, les émeutes françaises de 2005 ont été présentées par les médias comme des «muslim riots». 
alors une série de questionnements. Quels sont les auteurs traduits? Dans quels pays et de quelle manière les traductions influencentelles la compréhension et la représentation des banlieues hors de France? Préfère-t-on une traduction étrangéisante qui préserve les realia ou une adaptation transculturelle? Les éditeurs demandentils au traducteur d'être "transparent», pour employer les termes de Venuti, ou sollicitent-ils la présence d'un apparat paratextuel, selon une modalité de traduction qu'Appiah qualifierait de «dense»? Et encore, qui choisit ces textes (les éditeurs, les traducteurs, les universitaires...), et quel accueil leur réserve-t-on? Autant de questions qui illustrent les problématiques et les débats auxquels sont confrontés les chercheurs dans ce champ encore à défricher.

Pour éviter que ces questions demeurent un écheveau inextricable, les chercheurs qui s'engagent dans ce domaine doivent mettre en jeu une multiplicité d'approches qui ne viennent pas uniquement des théories «occidentales» de la traductologi $\mathrm{e}^{11}$; ils doivent accepter des solutions parfois provisoires et souvent inattendues. Afin d'aborder le sujet dans toute sa complexité, cette livraison de $T T R$ rassemble des études qui discutent de définitions théoriques, de grilles de lecture et d'outils traductionnels. Le théorique s'imbrique au pragmatique, pour souligner la valeur heuristique de l'acte traductif. Les spécialistes qui ont contribué à ce volume, sociolinguistes, traducteurs et traductologues versés dans l'étude de l'extrême contemporain, sont issus de formations et de pays divers. Leurs perspectives et leurs démarches reflètent leurs parcours et les enjeux auxquels ils s'intéressent.

Béatrice Turpin ouvre ce numéro en posant la question de l'éditorialisation des ouvrages de fiction qui ont au cœur la banlieue. Reconnue pour ses études des discours sociaux et médiatiques, elle nous invite à réfléchir à l'un des aspects paratextuels primordiaux dans le processus éditorial : le choix de la couverture. Turpin explique comment les publications françaises affichent la prédominance d'un point de vue réaliste, la couverture pouvant être considérée comme «image arrêtée d'un scénario». Les éditions traduites tendent, en revanche, à élargir le champ du regard en contextualisant le cadre

11. On ne soulignera jamais assez l'importance de la contribution des traductologues du «virage postcolonial» dans notre champ d'études, notamment celle de traductologues non occidentaux comme Paul Bandia et Kwame Anthony Appiah. Ajoutons aussi, en passant, que la littérature qui traite des banlieues françaises est bien plus étudiée à l'étranger qu'en France (Horvath, 2016, p. 47). 
historique ou spatial du récit ou, au contraire, en effaçant celui-ci, selon le pays et le contexte éditorial. La réflexion de Turpin, au-delà de sa prégnance spécifique, nous invite à considérer l'importance du positionnement de l'éditeur sur l'échiquier éditorial, notamment dans le cas de romans qui abordent des sujets délicats comme celui des banlieues contemporaines.

Dans la contribution suivante, Pierre-Alexis Mével nous fait pénétrer plus loin dans le domaine en question. L'auteur nous offre une réflexion stimulante sur l'oralité dans le texte littéraire, en focalisant son analyse sur le roman Allah Superstar (2003) d'Y. B. Son étude met savamment en jeu ses connaissances de la traduction audiovisuelle (notamment des «hood films» et des "films de banlieues»), pour voir de quelle manière l'oralité représentée dans ce roman, véritable stand-up d'un jeune banlieusard d'origine algérienne, peut être reproduite par le traducteur. Après une analyse détaillée des procédés exploités par Y. B., Mével suggère des pistes potentielles pour le traducteur anglais, pistes qui pourraient s'appliquer, toute proportion faite, à d'autres langues-cultures cibles. Au-delà d'une remise en cause salutaire de la dichotomie «domestication»/«foreignization», sa contribution nous fait réfléchir aux rapports étroits entre littérature et cinéma ${ }^{12}$, et à ce que la réflexion traductologique du domaine de l'audiovisuel peut apporter au champ de la traduction littéraire.

Larticle de Katrien Lievois, Nahed Nadia Noureddine et Hanne Kloots se concentre sur la littérature post-émeutes 2005, et particulièrement sur un corpus de traductions en arabe, espagnol et néerlandais du roman Kiffe kiffe demain (2004) de Faïza Guène. Ce roman, qui demeure l'un des plus traduits de notre champ de recherche (plus d'une vingtaine de langues), se prête particulièrement bien à une réflexion traductologique. Les chercheuses ont isolé vingt et un mots appartenant à la langue des jeunes des cités et analysé les stratégies adoptées par les traducteurs dans soixantedeux passages du roman dans lesquels figurent ces mots. L'étude des

12. Il s'agit d'un couple bien assorti et assez fréquent dans notre domaine. Il n'est pas rare que les écrivains de notre corpus soient aussi des réalisateurs (on pense, entre autres, à Samuel Benchetrit, Rachid Djaïdani et Faïza Guène) et qu'ils créent ainsi une poétique transmédiale (à ce sujet, Impellizzeri [2018] parle d'une «banlieuepoétique»). Les spécialistes de l'extrême contemporain savent d'ailleurs à quel point littérature et cinéma sont de plus en plus imbriqués l'un dans l'autre par des procédés tels que l'écriture scénaristique et la novellisation. 
traductions arabe, néerlandaise et espagnole de Kiffe kiffe demain montre un penchant pour la standardisation de la langue des jeunes des cités, bien que les trois spécialistes relèvent des distinctions entre les stratégies appliquées par les traducteurs pour les trois langues-cultures prises en compte.

Mattias Aronsson prolonge les réflexions théoriques autour du roman de Faïza Guène, sans aucun doute le plus étudié par la critique universitaire cette dernière décennie. Il analyse Kiffe kiffe demain sous un autre jour, celui de la réception, en se focalisant sur le contexte suédois. Le chercheur s'interroge ici sur les comptes rendus publiés dans la presse et sur Internet entre 2006 (année de la première traduction de Guène en suédois) et 2016. D'après Aronsson, les journalistes et les blogueurs s'attardent sur l'humour de l'auteure ainsi que sur une réflexion générale autour du milieu socioculturel de la banlieue défavorisée. Du point de vue du positionnement dans le champ littéraire, les journalistes suédois associent l'œuvre de Guène aux genres de la littérature migrante et, à un moindre degré, de la littérature prolétarienne. Le style de l'auteure, truffé d'argotismes, est quant à lui apprécié différemment et constitue un sujet clivant.

Avec la contribution suivante, nous entrons dans le champ de la lexicographie. Evangelos Kourdis a en effet choisi de se pencher sur un corpus de quatre dictionnaires argotiques, qu'il analyse depuis une perspective intersémiotique. Le spécialiste montre de quelle manière l'illustration devient un dispositif de médiation pour le lecteur francophone, la traduction intersémiotique aidant le lecteur du dictionnaire à dépasser certains obstacles de compréhension concernant le vocabulaire argotique. D'après Kourdis, l'illustration peut être un moyen d'explication, mais elle pose également des limites à la vulgarisation de certains lexèmes. Dans son étude, l'auteur montre de quelle manière l'illustration dans les dictionnaires argotiques - outils indispensables pour les traducteurs qui abordent notre champ de recherche - est appelée à jouer un rôle crucial dans l'orientation sémantique, car elle peut coopérer (ou non) à la consolidation des lexèmes dans la mémoire culturelle individuelle et collective.

Matt Reeck nous propose pour sa part une réflexion originale autour de l'œuvre de Rachid Djaïdani, l'un des écrivains le plus intéressants de notre champ d'études. Reeck décrit comment l'auteur adopte la perspective d'un traducteur-interprète ethnologue dans 
son premier roman, Boumkøur (1999). Décrivant la banlieue française comme un terrain d'étude ethnographique, Djaïdani montre de quelle manière son héros, Yaz, en tant que narrateurethnographe, prend part à des tâches de traduction d'ordre non seulement linguistique, mais culturel. Échappant à un horizon d'attente stéréotypé, le roman propose une ethnographie littéraire expérimentale, conçue comme une forme nouvelle de traduction, dans laquelle le traducteur assume une position active dans le texte et aide à établir la traduction comme acte de négociation. La force de l'approche de Reeck réside dans la mise en résonance des enjeux de l'ethnographie et de la traductologie, intersection que d'autres chercheurs, dont Sherry Simon, ont aussi examinée.

L'article signé par Satoshi Udo offre une perspective inédite sur la traduction et la réception de la banlieue française au Japon. L'auteur y dévoile avec finesse l'évolution de la terminologie et de la conception de la banlieue française au pays du Soleil Levant, et comment elles ont modelé la perception de la périphérie japonaise et de sa représentation littéraire. En suivant une approche diachronique, Udo promène son regard sur deux romans dans un aller-retour constant entre Orient et Occident: Vers la banlieue (1995) d'Horie Toshiyuki, auteur japonais et traducteur de littérature française, qui raconte la banlieue de Paris par des impressions de voyage et des détours livresques, et Le petit Malik (2008) de Mabrouck Rachedi, auteur français publié au Japon avec un riche apparat paratextuel, qui met en évidence le côté sociologique du milieu suburbain où se situe la narration. Entre ces deux visions très différentes non seulement de la périphérie, mais aussi des voix des marges et de leur traduction possible, la banlieue demeure aux yeux des Japonais un lieu en devenir.

Dans l'article qui clôt ce numéro, je présente une analyse des défis traductologiques posés par la littérature qui met en scène la banlieue, que je regroupe en deux catégories interdépendantes: les obstacles internes, liés à l'emploi créatif de l'argot des cités par les auteurs, et les obstacles externes, qui concernent les politiques éditoriales ainsi que les facteurs sociaux et économiques liés à la circulation des œuvres dans le marché contemporain. En prenant appui sur les travaux de Gisèle Sapiro, je développe une étude sociotraductologique en suivant la traduction italienne des romans de Saphia Azzeddine, du choix éditorial jusqu'à leur diffusion. 
En plus d'illustrer mes propres outils conceptuels et mes choix traductionnels, j’essaie de montrer les défis, les obstacles et les aléas qui caractérisent le processus éditorial.

Les articles réunis dans cette livraison offrent, dans leur ensemble, une variété d'approches et dessinent un panorama critique sur un sujet qui demeure encore peu étudié. Ils discutent des positions qui peuvent être adoptées par les traducteurs et par les éditeurs, en soulignant, entre autres, la nécessité d'une remise en question des modèles épistémologiques strictement binaires (on pense à la dichotomie souvent abusive entre "domestication» et «foreignization»), qui s'appliquent mal à des auteurs qui font de l'hybridation, de l'hétéroglossie et du «multilinguisme littéraire» (Stratford, 2008) leur bannière, et qui essaient de sortir des oppositions manichéennes entre centre et périphérie en remettant en question leurs polarités. La réflexion traductologique proposée autour de ces textes difficiles à classer et qui échappent de plus en plus aux discours dualistes saura certainement inspirer de nouvelles pistes traductives et traductologiques, non seulement en contribuant à l'adoption d'approches inédites pour la traduction de la "parole des marges» - et particulièrement des textes littéraires dont la langue s'aventure hors des sentiers battus -, mais aussi en participant à un débat théorique actuel bien plus large.

\section{Références}

Appiah, Kwame Anthony (1993). «Thick Translation». Callaloo, 16, 4, p. 808-819.

Bertucci, Marie-Madeleine et Daniel Delas, dir. (2004). Français des banlieues, français populaire? Amiens, Ancrage.

Bourdieu, Pierre (2002). «Les conditions sociales de la circulation internationale des idées». Actes de la recherche en sciences sociales, 145, p. 3-8.

Deleuze, Gilles et Félix Guattari (1975). Kafka. Pour une littérature mineure. Paris, Minuit.

Djaïdani, Rachid (2007). Viscéral. Paris, Seuil.

Even-Zohar, Itamar (1990). «Polysystem Studies». Poetics Today, 11, 1.

Gadet, Françoise, dir. (2017). Les parlers jeunes dans l'Île-de-France multiculturelle. Paris, Ophrys.

Goudaillier, Jean-Pierre (1997). Comment tu tchatches! Dictionnaire du français contemporain des cités. Paris, Maisonneuve et Larose.

Haas, Valérie et Capucine Vermande (2010). «Les enjeux mémoriels du passé colonial français : analyse psychosociale du discours de la presse, lors des émeutes urbaines de novembre $2005 »$. Bulletin de psychologie, 63, 2, p. 109-120. 
Hargreaves, Alec G. (2014). «De la littérature "beur" à la littérature de "banlieue" : des écrivains en quête de reconnaissance». Africultures, 97, p. 144-149.

Hargreaves, Alec G. (2016). "Banlieue, islam: une médiatisation qui dérange». In B. Wallon, dir. Banlieues vues d'ailleurs, Paris, CNRS Éditions, p. 83-100.

Horvath, Christina (2016). «Écrire la banlieue dans les années 2000$2015 »$. In B. Wallon, dir. Banlieues vues d'ailleurs, Paris, CNRS Éditions, p. 47-68.

Impellizzeri, Fabrizio (2018). «Des mots du bitume au maux de l'asphalte. L'auto-adaptation cinématographique de la "banlieuepoétique" de Samuel Benchetrit». In I. Vitali, dir. Banlieues en textes: traduction, adaptation, réception, Atelier de traduction, 29, p. 129-144.

Kasbarian, Jean-Michel (1997). «Quelques repères pour décrire le langage des banlieues». Actes du colloque Touche pas à ma langue! Les langages de banlieue, Skholê, Cahiers de la recherche et du développement, numéro hors-série, p. 23-39.

Kokoreff, Michel et Didier Lapeyronnie (2013). Refaire la cité. L'avenir des banlieues. Paris, Éditions du Seuil et La République des Idées.

Lapeyronnie, Didier (2005). «La banlieue comme théâtre colonial, ou la fracture coloniale dans les quartiers». In P. Blanchard, N. Bancel et S. Lemaire, dir. La fracture coloniale. La société française au prisme de l'héritage colonial. Paris, La Découverte, p. 209-218.

Lepape, Pierre (2003). Le pays de la littérature. Des Serments de Strasbourg à l'enterrement de Sartre. Paris, Seuil.

Lepoutre, David (1997). Cceur de banlieue. Paris, Odile Jacob.

Paquot, Thierry, dir. (2008). Banlieue. Une anthologie. Lausanne, Presses polytechniques et universitaires romandes.

Puig, Stève (2011). «Du roman beur au roman urbain: de L'intégration d'Azouz Begag à Désintégration d'Ahmed Djouder». In I. Vitali, dir. Intrangers. De l'écriture à la traduction, Louvain-la-neuve, Academia Bruylant, p. 21-46.

Qui fait la France? (2007). Chroniques d'une société annoncée. Paris, Stock.

Reeck, Laura (2011). Writerly Identities in Beur Fiction and Beyond. Lanham, Lexington Books.

Reeck, Laura et Kathryn Kleppinger (2018). Post-migratory Cultures in Postcolonial France. Liverpool, Liverpool University Press.

Sapiro, Gisèle, dir. (2012). Traduire la littérature et les sciences humaines. Conditions et obstacles. Paris, La documentation française.

Sherry, Simon, dir. (2005). La traduction engagée. TTR, 18, 2.

Stratford, Madeleine (2008). «Au tour de Babel! Les défis multiples du multilinguisme». Meta, 53, 3, p. 457-727.

Toury, Gideon (1995). Descriptive Translation Studies and Beyond. Amsterdam/Philadelphia, John Benjamins. 
Venuti, Lawrence (1998). The Scandals of Translation: Towards an Ethics of Difference. New York, Routledge.

Vieillard-Baron, Hervé (1996). Les banlieues. Paris, Flammarion.

Vieillard-Baron, Hervé (2001). Les banlieues. Des singularités françaises aux réalités mondiales. Paris, Hachette.

Vieillard-Baron, Hervé (2008). «La banlieue : question de définition». In T. Paquot, dir. Banlieue. Une anthologie. Lausanne, Presses polytechniques et universitaires romandes.

Vitali, Ilaria, dir. (2011). Intrangers, I, Post-migration et nouvelles frontières de la littérature beur; Intrangers, II, Littérature beur: de l'écriture à la traduction. Louvain-la-Neuve, Academia Bruylant; Paris, L'Harmattan.

Vitali, Ilaria (2014). La nebulosa beur. Scrittori di seconda generazione tra spazio francese e letteratura-mondo. Bologna, Odoya/I libri di Emil.

Vitali, Ilaria (2015). «En long, en large et en verlan : l'emploi littéraire du français contemporain des cités». In F. Impellizzeri, dir. Les variations linguistiques dans la littérature et le cinéma contemporains. Paris, Classiques Garnier.

Wallon, Bernard, dir. (2016). Banlieues vues d'ailleurs. Paris, CNRS Éditions. 